\title{
A Case of Immune Thrombocytopenia as a Rare Side Effect of an Immunotherapy with PD1-Blocking Agents for Metastatic Melanoma
}

\author{
Claudia Pföhler ${ }^{a}$ Hermann Eichler ${ }^{b}$ Barbara Burgard ${ }^{a}$ Nathalie Kreckéa \\ Cornelia S.L. Müller ${ }^{a}$ Thomas Vogt $^{a}$ \\ ${ }^{a}$ Department of Dermatology, Saarland University Medical School, Homburg/Saar, Germany; \\ b Institute of Clinical Hemostaseology and Transfusion Medicine, Saarland University Medical School, Homburg/Saar, Germany
}

\section{Keywords}

Nivolumab · Pembrolizumab · Checkpoint blockade ·

Melanoma $\cdot$ Immune thrombocytopenia

\section{Summary}

Background: Checkpoint blocking agents such as pembrolizumab or nivolumab may induce a diversity of mostly autoimmune-mediated side effects. These autoimmune phenomena mainly affect ductless glands such as the pituitary gland (hypophysitis), the thyroid gland (thyreoiditis), the skin (vitiligo and rash), the colon (colitis), and the lung (pneumonitis). Furthermore, many other organs or organ systems may be affected. Case Report: This work describes a case of an immune thrombocytopenia that developed or rather became clinically significant shortly after initiation of a systemic therapy with first nivolumab and later pembrolizumab given due to metastatic melanoma. Platelet counts before this systemic therapy were slightly decreased with values around 110/nl (normal value $140-400 / n l$ ). Thrombocytopenia developed or became apparent rapidly within 10 days after the first intravenous application of nivolumab and worsened after changeover to pembrolizumab. Therapy had to be stopped due to disease progression and steady aggravation of thrombocytopenia. Immune hematology assays could prove an autoimmune mediated genesis of thrombocytopenia. Conclusion: Checkpoint inhibitors may induce a multiplicity of mostly autoimmune-mediated side effects. In contrast to chemotherapy-induced cytopenia that results from bone marrow toxicity, thrombocytopenia in melanoma patients treated with checkpoint inhibiting substances seems to result from autoimmune-mediated side effects in the majority of the cases. Thorough labora- tory controls during these therapies are therefore required. In case of thrombocytopenia, immune hematology testing to diagnose or rule out immune thrombocytopenia is indispensable.

(c) 2017 S. Karger GmbH, Freiburg

\section{Introduction}

Immune checkpoint blocking agents such as the CTLA-4 antibody ipilimumab or the PD1-antagonists pembrolizumab and nivolumab have been approved for the treatment of metastatic melanoma [1-4]. Ipilimumab blocks the CTLA-4 receptor on T cells and releases the breaks of the immune system by turning off inhibitory mechanisms in cytotoxic T cells. Pembrolizumab and nivolumab block binding of programmed death protein 1 receptor (PD1) on $\mathrm{T}$ cells and programmed death-ligand 1 (PDL1) on melanoma cells. Hence, due to the mode of action of these antibodies tumor-specific T-cell responses are restored. For ipilimumab as well as for both PD1 blockers, benefits in response rates as well as in progressionfree and overall survival were proven in the past [1-4]. However, all of these drugs may induce autoimmune phenomena such as thyroid dysfunction, vitiligo, rash, autoimmune colitis, or pneumonitis [5, 6]. Cases of severe forms of autoimmune-mediated thrombocytopenia have been reported in singular cases only [7-10].

\section{Case Report}

We report the case of a 73-year-old male patient with a V600E BRAF-mutated metastatic melanoma. The malignancy had been revealed in March 2015 by axillary metastases of a malignant melanoma without known primary. After complete resection of all tumor masses, an adjuvant radiotherapy of axillary,

\section{KARGER}

() 2017 S. Karger GmbH, Freiburg

Fax +497614520714 


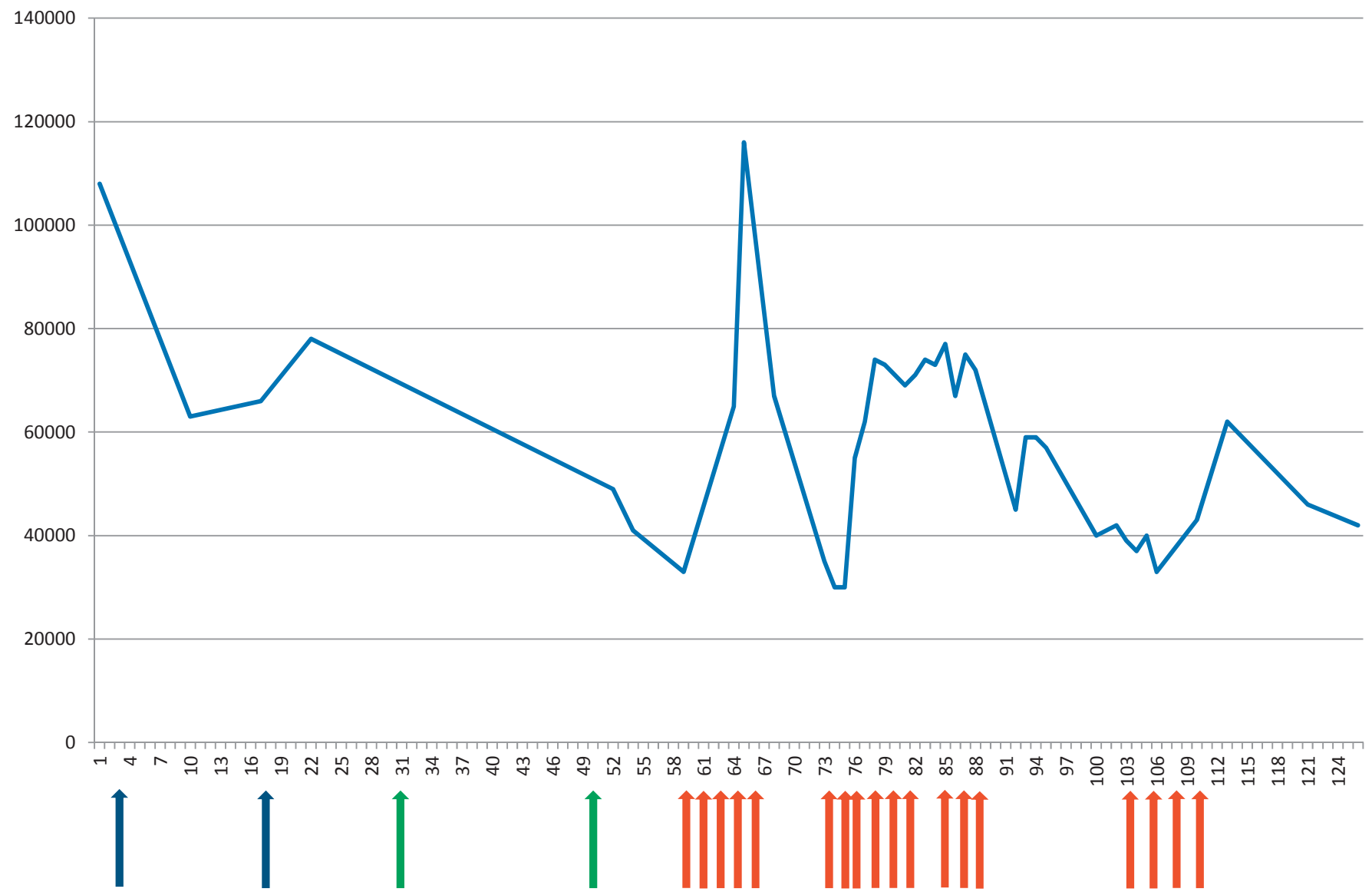

Fig. 1. Platelet counts during and after treatment with PD-1 blockers. Nivolumab was administered on day 3 and 17 (blue arrows); pembrolizumab was given on

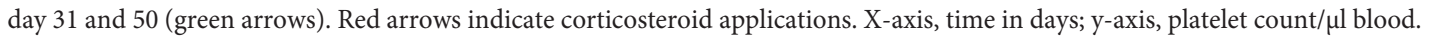

supra- and infraclavicular lymph nodes followed by an adjuvant immunotherapy with low-dose IFN- $\alpha$ was performed until December 2014. Under therapy with IFN- $\alpha$ Hashimoto thyreoiditis developed and was treated with orally administered thyroxin. In December 2014 disease progression occurred with disseminated lung, skin, lymph node, and bone metastases. The patient was first treated with the BRAF inhibitor LGX 818 within a clinical trial. Best response was regressive disease in April 2015, but due to disease progression (progressive disease in all metastatic sites) treatment had to be changed in December 2015. Platelet counts before and during this systemic therapy were slightly decreased with values around $130 / \mathrm{nl}$ (normal value $140-400 / \mathrm{nl}$ ). To treat aching progressive bone metastases, a radiation of the left pelvis and hip was performed in December 2015 with a cumulative dose of $33 \mathrm{~Gy}$. Then, a therapy with nivolumab ( $3 \mathrm{mg}$ every 2 weeks) was started in the end of December 2015. Baseline blood tests were normal except of a mild thrombocytopenia $(108 / \mathrm{nl})$ that had been interpreted as a side effect of radiation. The first dose of nivolumab was well tolerated without any side effects. The application of the second dose led to an anaphylactic reaction presenting with generalized urticaria, hypotension, and tachycardia approximately half an hour after infusion completion. Therefore, in January 2016 treatment was changed to pembrolizumab with a dose of $2 \mathrm{mg} / \mathrm{kg}$ every 3 weeks after premedication with dexamethasone $8 \mathrm{mg}$, ranitidine $300 \mathrm{mg}$ and clemastine $2 \mathrm{mg}$ intravenously. Treatment was tolerated without any subjective side effects. In February 2016 therapy was stopped due to further disease progression with development of multiple brain metastases and worsened thrombocytopenia. Performing immune hematology testing in March 2016 using serum and platelets of the patient for the platelet immune fluorescent assay (PIFA) and the monoclonal antibody-specific immobilization of platelet antigen assay (MAIPA), free and cell-bound platelet-specific IgG autoantibodies could be detected $[11,12]$. In detail, in two independent indirect PIFA assays on March 2 and 24 , a relative fluorescence intensity of $43.8 \%$ and $48.0 \%$ was detected, re- spectively (cut-off $>20 \%$, negative controls $1.8 \%$ and $2.3 \%$ ). The direct PIFA on March 24 proved that the patient's platelets were strongly covered with IgG autoantibodies (relative fluorescence intensity $96.2 \%$, cut-off $>20 \%$, negative control 13.8\%). Moreover, performing the indirect MAIPA with the blood sample of March 2, neither alloantibodies against platelet-specific glycoproteins nor HLAspecific alloantibodies could be detected. Due to the clinical course and the laboratory results, it is likely that the thrombocytopenia is caused by an autoimmune-mediated genesis. However, other possible causes of thrombocytopenia were not excluded, e.g. by performing a bone marrow aspirate. Systemic corticosteroids were administered (first in a dose of $1 \mathrm{mg}$ methylprednisolone/kg body weight, then tapered down). However, platelet counts returned to values around 130/nl not before July 2016. Figure 1 shows platelet count and therapy of autoimmune-mediated thrombocytopenia. Due to disease progression, a wholebrain radiation was performed with a cumulative dose of $36 \mathrm{~Gy}$, followed by targeted therapy with dabrafenib and trametinib that led to a short-lasting partial response. The patient died due to metastatic disease in September 2016.

\section{Discussion}

Autoimmune thrombocytopenia can be classified into a primary or a secondary form, dependent on the absence or presence of associated or underlying diseases [13]. The secondary form may be associated with infections, e.g. HCV or HIV, malignant tumors, e.g. lymphoma or solid tumors, or with autoimmune diseases, e.g. Evans syndrome or lupus erythematodes [13]. Aboud and coworkers [13] could show in a large retrospective analysis that $11 \%$ of 
patients with autoimmune thrombocytopenia suffered from affections of the thyroid gland just as well as the patient presented here who had developed Hashimoto thyreoiditis under therapy with IFN- $\alpha$ in the past. In general, autoimmune disorders tend to coexist in the same individual [14]. Boelaert et al. [15] could show that patients with Hashimoto thyreoiditis suffered from another autoimmune disorder in $14.3 \%$. The relative risk to develop another autoimmune disorder such as pernicious anemia, systemic lupus erythematodes, Addison's disease, celiac disease, or vitiligo was more than 10 -fold elevated in patients with Hashimoto thyreoiditis [15]. Whether the patient presented here suffered from immune thrombopenia before treatment with checkpoint inhibitors or not must remain speculative as laboratory testing was not performed at that point in time. Though, a preexisting condition seems to be most likely because platelet count was slightly decreased with values around 110/nl before immunotherapy with PD1 blockers. In any case, therapy with nivolumab and pembrolizumab aggravated thrombopenia and induced or rather increased the production of platelet-specific IgG autoantibodies. Due to this clinical course and the laboratory results, it is likely that the thrombocytopenia was caused by PD-1 inhibitor-induced platelet autoantibodies via an autoimmunity activation. However, other possible causes of thrombocytopenia were not excluded in this case. Therefore, the final prove remains open.

To date, only a few cases of immune thrombopenia resulting from therapy with checkpoint inhibitors have been published [710]. However, in large prospective clinical trials investigating checkpoint inhibitors in different malignancies, 'cytopenia' was reported with an incidence between 5 and 17\% [9]. Regrettably, the genesis of thrombocytopenia has not been clarified in these studies. Yet, it can be presumed that in a large part of cases with thrombopenia an autoimmune-mediated pathomechanism was causative.

In summary, the incidence of immune thrombopenia in tumor patients treated with checkpoint inhibitors is potentially much higher than previously suspected. Meticulous controls of blood count are therefore essential. In cases of thrombopenia one should bear in mind that low or declining platelet counts could be a sign of immune thrombopenia.

\section{Ethical Approval}

Written informed consent from the patient was obtained.

\section{Acknowledgments}

The authors are very grateful to the patient and his family for their kind support of this case report.

\section{Disclosure Statement}

CP received honoraria and travel support from MSD Sharp \& Dohme and Bristol-Myers Squibb. All other authors have nothing to declare.

\section{References}

1 Robert C, Long GV, Brady B, Dutriaux C, Maio M, Mortier L, Hassel JC, Rutkowski P, McNeil C, KalinkaWarzocha E, Savage KJ, Hernberg MM, Lebbe C, Charles J, Mihalcioiu C, Chiarion-Sileni V, Mauch C, Cognetti F, Arance A, Schmidt H, Schadendorf D, Gogas H, Lundgren-Eriksson L, Horak C, Sharkey B, Waxman IM, Atkinson V, Ascierto PA: Nivolumab in previously untreated melanoma without BRAF mutation. N Engl J Med 2015;372:320-330.

2 Robert C, Ribas A, Wolchok JD, Hodi FS, Hamid O, Kefford R, Weber JS, Joshua AM, Hwu WJ, Gangadhar TC, Patnaik A, Dronca R, Zarour H, Joseph RW, Boasberg P, Chmielowski B, Mateus C, Postow MA, Gergich K, Elassaiss-Schaap J, Li XN, Iannone R, Ebbinghaus SW, Kang SP, Daud A: Anti-programmed-deathreceptor-1 treatment with pembrolizumab in ipilimumab-refractory advanced melanoma: a randomised dose-comparison cohort of a phase 1 trial. Lancet 2014;384:1109-1117.

3 Robert C, Schachter J, Long GV, Arance A, Grob JJ, Mortier L, Daud A, Carlino MS, McNeil C, Lotem M, Larkin J, Lorigan P, Neyns B, Blank CU, Hamid O, Mateus C, Shapira-Frommer R, Kosh M, Zhou H, Ibrahim N, Ebbinghaus S, Ribas A, investigators K-: Pembrolizumab versus ipilimumab in advanced melanoma. N Engl J Med 2015;372:2521-2532.

4 Weber JS, D'Angelo SP, Minor D, Hodi FS, Gutzmer R, Neyns B, Hoeller C, Khushalani NI, Miller WH, Jr., Lao CD, Linette GP, Thomas L, Lorigan P, Grossmann KF, Hassel JC, Maio M, Sznol M, Ascierto PA, Mohr P, Chmielowski B, Bryce A, Svane IM, Grob JJ, Krackhardt AM, Horak C, Lambert A, Yang AS, Larkin J: Nivolumab versus chemotherapy in patients with advanced melanoma who progressed after anti-CTLA-4 treatment (CheckMate 037): a randomised, controlled, open-label, phase 3 trial. Lancet Oncol 2015;16:375-384.

5 Hofmann L, Forschner A, Loquai C, Goldinger SM, Zimmer L, Ugurel S, Schmidgen MI, Gutzmer R, Utikal JS, Goppner D, Hassel JC, Meier F, Tietze JK, Thomas I, Weishaupt C, Leverkus M, Wahl R, Dietrich U, Garbe C, Kirchberger MC, Eigentler T, Berking C, Gesierich A, Krackhardt AM, Schadendorf D, Schuler G, Dummer R, Heinzerling LM: Cutaneous, gastrointestinal, hepatic, endocrine, and renal side-effects of anti-PD-1 therapy. Eur J Cancer 2016;60:190-209.

6 Zimmer L, Goldinger SM, Hofmann L, Loquai C, Ugurel S, Thomas I, Schmidgen MI, Gutzmer R, Utikal JS, Goppner D, Hassel JC, Meier F, Tietze JK, Forschner A, Weishaupt C, Leverkus M, Wahl R, Dietrich U, Garbe C, Kirchberger MC, Eigentler T, Berking C, Gesierich A, Krackhardt AM, Schadendorf D, Schuler G, Dummer R, Heinzerling LM: Neurological, respiratory, musculoskeletal, cardiac and ocular side-effects of anti-PD-1 therapy. Eur J Cancer 2016;60:210-225.

7 Inadomi K, Kumagai H, Arita S, Tsuruta N, Takayoshi K, Mishima K, Ota S, Tanaka M, Okumura Y, Sagara K, Nio K, Nakano M, Uchi H, Yamamoto H, Ariyama H, Kusaba H, Niiro H, Oda Y, Akashi K, Baba E: Bicytopenia possibly induced by anti-PD-1 antibody for primary malignant melanoma of the esophagus: a case report. Medicine (Baltimore) 2016;95:e4283.

8 Kanameishi S, Otsuka A, Nonomura Y, Fujisawa A, Endo Y, Kabashima K: Idiopathic thrombocytopenic purpura induced by nivolumab in a metastatic mela- noma patient with elevated PD-1 expression on B cells. Ann Oncol 2016;27:546-547.

9 Le Roy A, Kempf E, Ackermann F, Routier E, Robert C, Turpin A, Marabelle A, Mateus C, Michot JM, Lambotte O: Two cases of immune thrombocytopenia associated with pembrolizumab. Eur J Cancer 2016;54:172-174.

10 Solomon LR: Thrombocytopenia due to low-dose colchicine therapy: a possible drug interaction with nivolumab and implications for supportive care. Acta Oncol 2015;54:1235-1237.

11 Hagenstrom H, Schlenke P, Hennig H, Kirchner H, Kluter H: Quantification of platelet-associated IgG for differential diagnosis of patients with thrombocytopenia. Thromb Haemost 2000;84:779-783.

12 Kiefel V, Santoso S, Weisheit M, Mueller-Eckhardt C: Monoclonal antibody-specific immobilization of platelet antigens (MAIPA): a new tool for the identification of platelet-reactive antibodies. Blood 1987;70:1722-1726.

13 Aboud N, Depré F, Salama A: Is autoimmune thrombocytopenia itself the primary disease in the presence of second disease? Data from a long-term observation. Transfus Med Hemother 2017;44:23-28.

14 Ponto KA, Schuppan D, Zwiener I, Binder H, Mirshahi A, Diana T, Pitz S, Pfeiffer N, Kahaly GJ: Thyroid-associated orbitopathy is linked to gastrointestinal autoimmunity. Clin Exp Immunol 2014;178:57-64.

15 Boelaert K, Newby PR, Simmonds MJ, Holder RL, CarrSmith JD, Heward JM, Manji N, Allahabadia A, Armitage M, Chatterjee KV, Lazarus JH, Pearce SH, Vaidya B, Gough SC, Franklyn JA: Prevalence and relative risk of other autoimmune diseases in subjects with autoimmune thyroid disease. Am J Med 2010;123:183.e1-9. 